\title{
PUBLISHER CORRECTION TO: POINT-LIKE BOUNDING CHAINS IN OPEN GROMOV-WITTEN THEORY
}

\author{
Jake P. Solomon and Sara B. Tukachinsky
}

\section{Publisher Correction to: Geom. Funct. Anal. https://doi.org/10.1007/s00039-021-00583-3}

Due to a processing error, the affiliations of authors were published incorrectly. The correct affiliations are:

Institute of Mathematics, Hebrew University, 9190401, Givat Ram, Jerusalem, Israel Jake P. Solomon

School of Mathematical Sciences, Tel Aviv University, 6997801, Tel Aviv, Israel Sara B. Tukachinsky

We sincerely apologize for any inconvenience.

The Publisher

Publisher's Note Springer Nature remains neutral with regard to jurisdictional claims in published maps and institutional affiliations.

J. P. SOLOMON

Institute of Mathematics, Hebrew University, 9190401 Givat Ram, Jerusalem, Israel.

S. B. TUKACHINSKY

jake@math.huji.ac.il

School of Mathematical Sciences, Tel Aviv University, 6997801 Tel Aviv, Israel.

sarabt1@gmail.com

The original article can be found online at https://doi.org/10.1007/s00039-021-00583-3 